\title{
Evaluating potential threats to birds in Greece: an analysis of a 10-year data set from a rehabilitation centre
}

\author{
Antonios D. Mazaris, Yiannis Mamakis, Stauros Kalpakis \\ Yiannis Poulopoulos and Yiannis G. Matsinos
}

\begin{abstract}
In an attempt to identify the critical threats to avian biodiversity in Greece we present and analyse a data set of the birds received at a rehabilitation centre. During a 10-year period (1996-2005) a total of 21,190 birds were admitted to the Hellenic Wildlife Hospital from throughout Greece. Our results indicate that shooting is by far the most important threat to birds in Greece, followed by accidents. Although greater numbers of shot birds were received during the autumn and winter months, when hunting is allowed, birds were received year-round. Birds were received from all areas of Greece, with the highest number received during the summer months. The high number of shot birds received during the months in which hunting is prohibited demonstrates the lack of law enforcement. We argue the need for direct political decisions concerning the enforcement of existing regulations on hunting. Decision makers and managers could use the findings of our study to re-evaluate and design conservation policy in Greece, including reduction of the hunting period and restriction on hunting activities.
\end{abstract}

Keywords Bird conservation, Greece, hunting, mortality, potential threats, rehabilitation centre, shooting.

\section{Introduction}

$\mathrm{D}$ eclines in biological diversity have accelerated the need for efficient conservation plans (Pullin, 2002), and the determination of the main threats to wildlife is fundamental for the formulation of such plans (Soulé \& Orians, 2001). Quantitative assessment of threats is important for two main reasons: it improves our ability to protect wildlife and ecosystems according to their conservation status and requirements (Letcher et al., 1998; Wemmer et al., 2001; Sullivan et al., 2006; Leston et al., 2006), and helps sustain and justify calls for support and action to govern-

Antonios D. Mazaris (Corresponding author) Department of Ecology, School of Biology, Aristotle University, UPB 119, 54124 Thessalonica, Greece. E-mail amazar@bio.auth.gr

Yiannis Mamakis and Yiannis G. Matsinos Department of Environment, University of the Aegean, GR-81100 Myitilene, Greece.

Stauros Kalpakis and Yiannis Poulopoulos Hellenic Wildlife Hospital, Aigina, GR-18010, Greece.

Received 25 September 2006. Revision requested 15 January 2007. Accepted 30 April 2007. mental and international bodies (Johnson et al., 2005; Mishra et al., 2006).

The migratory nature of birds, with many species covering great distances in seasonal and reproduction migrations, and also during daily foraging, make it difficult to collect a comprehensive data set on threats. However, wildlife rehabilitation centres can provide information (International Wildlife Rehabilitation Council, 2007) that could also be used to improve our knowledge of factors affecting the viability and persistence of both species and populations (Rijksen, 1974; Barten, 1986; Underhill et al., 1999; Parsons et al., 2006). Data collected at rehabilitation centres include treatment, success of rehabilitation and post-release survival of wildlife (Clumper et al., 2000), especially with respect to treatment and rehabilitation success of wild animals affected by oil spills (MignucciGiannoni, 1999; Tseng, 1999; Parsons \& Underhill, 2005) and the rehabilitation of animals released after fire (Lunney et al., 2004).

Data on the main causes of morbidity and mortality of wildlife species is recorded by rehabilitation centres (Burton \& Dufour, 2000; Casey \& Casey, 2000). Studies that focus on particular groups (Miller, 1997; Deem et al., 1998; Morishita et al., 1998; Brown \& Sleeman, 2002), species of particular interest (Schulz, 1986; Porter \& McEntyre, 1988), and specific types of injuries or diseases (Jones, 1999) can help evaluate potential threats and population trends at local or regional scales (Casey \& Casey, 2000).

Here we use data collected over a 10-year period at the Hellenic Wildlife Hospital, Greece, to provide insights into the main threats to wild birds in the country. To our knowledge this is the first such study in the eastern Mediterranean. Greece, as a member of the European Union, has rights and obligations under international and European law, and our findings could help identify possible gaps in law enforcement or conservation efforts.

We have four main objectives: (1) To examine the numbers of birds admitted to the Hospital during a 10-year period, documenting the main reasons for entry and whether these causes of morbidity and mortality vary over time. (2) To examine seasonal patterns in numbers of birds admitted and reasons for admittance. (3) To examine and describe possible links between specific threats, species and areas by comparing records of birds received from the zoogeographical regions of Greece. (4) To study whether 
particular groups of species are more sensitive to specific threats.

\section{Methods}

\section{Data collection}

Data used in this study were collected during a 10-year period (1996-2005) from the Hellenic Wildlife Hospital, established in 1984 in Aigina, Greece. The centre receives birds, mammals, amphibians and reptiles from throughout Greece. All animals admitted receive a physical examination and individual records include the date and reason of admission, taxon, area of origin, and suggested treatment. In our analysis we classified the reasons for admission of birds as: (1) shooting, (2) poisoning/illness, (3) accident, (4) weakness, (5) orphan/newborn, (6) captivity. Accidents include electrocution, oiling and vehicle-caused injuries, weakness includes exhausted birds, and captivity includes illegally captured birds or abandoned pets.

\section{Data analysis}

To evaluate any differences among the birds received from different areas, we determined their distribution based on the 11 zoogeographical regions of Greece (Dafis et al., 1997; Fig. 1). Bird records were subdivided into raptors, aquatic and migratory. Species of conservation interest were determined based on the Birds Directive of the European Union 79/409/EEC; this Directive lists species that need protection and occur in the wild in European territory. Date of admission data were classified into four 3-month periods (autumn, September-November; winter, DecemberFebruary; spring, March-May; summer, June-August) to facilitate seasonal analysis. To interpret our findings with respect to hunting and non-hunting periods we assumed that autumn and winter are the hunting period and spring and summer the closed season. However, the opening and closing dates for hunting in Greece vary depending on species (starting dates 15 August-20 September; closing dates 30 October-28 February), and variations in these dates occur for the same species between years.

We used Kruskal-Wallis tests to examine differences in the number of birds admitted per season, from different zoogeographical regions, and per season according to the reasons for admittance, and the Nemenyi test for nonparametric multiple comparisons (Zar, 1999). We used a $\chi^{2}$ test for the hypothesis that the number of birds received per season is uniform among the 10 years of the study.

We used non-metric multidimensional scaling analysis (NMS) to examine the association among the 11 zoogeographical regions regarding the taxonomic composition of species received at the hospital. NMS is an ordination technique that attempts to explain the observed similarities or dissimilarities between the studied objects. All pairwise similarities or dissimilarities (distances) between the objects are expressed in correlation matrices that are then analysed

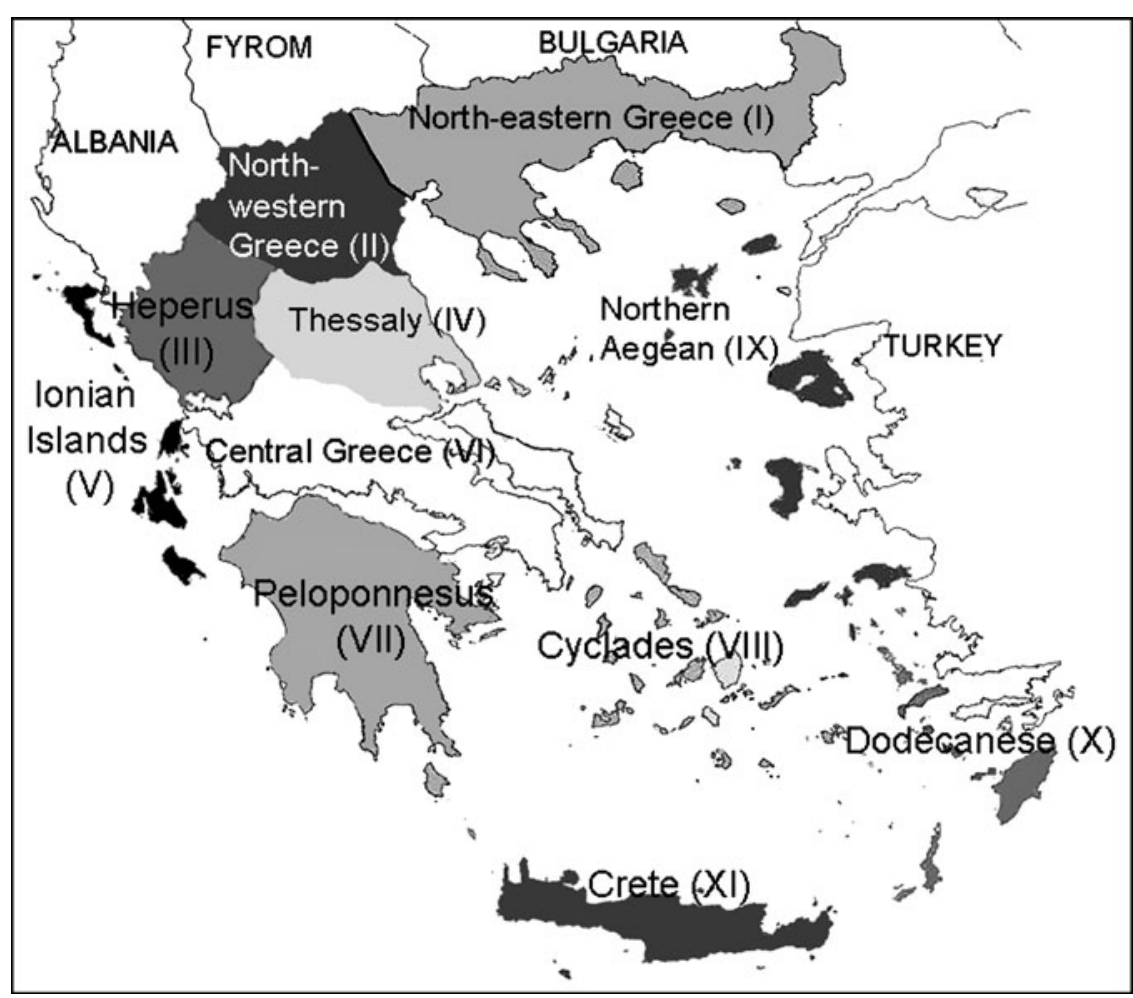

FIG. 1 Zoogeographical regions of Greece (based on Dafis et al., 1997, as modified by Kuehnelt, 1965, and Willemse, 1984). 


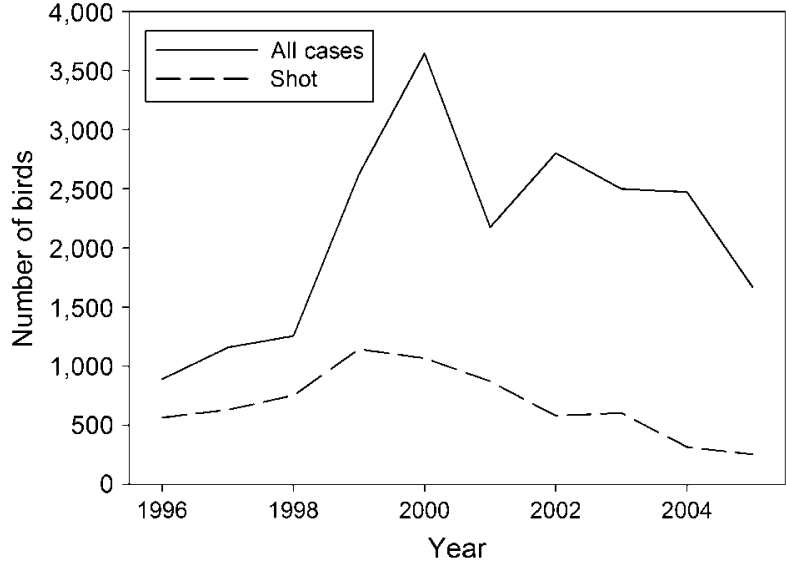

FIG. 2 Numbers of all birds and shot birds admitted each year over 1996-2005 to the Hellenic Wildlife Hospital.

to identify the orthogonal axes that are able to explain most of the variation. The depiction of those axes in ordination diagrams allows the visual detection and exploration of similarities or dissimilarities within the data. A goodness of fit test, the stress value, was used to assess whether the ordination plot reproduces the observed distance matrix successfully; lower values of stress demonstrate a better representation, with values $<0.1$ suggesting an acceptable configuration of the data. We performed a further NMS to investigate the relationships between the 11 zoogeographical regions based on the causes of mortality or morbidity. Prior to NMS analysis data were square root transformed. BrayCurtis similarity was used as a similarity measure. The minimum stress results were obtained after 100 iterations. NMS ordinations were performed using the PRIMER package (Clarke \& Gorley, 2001). Other statistical analyses were performed using SPSS v. 13 (SPSS, Chicago, USA).

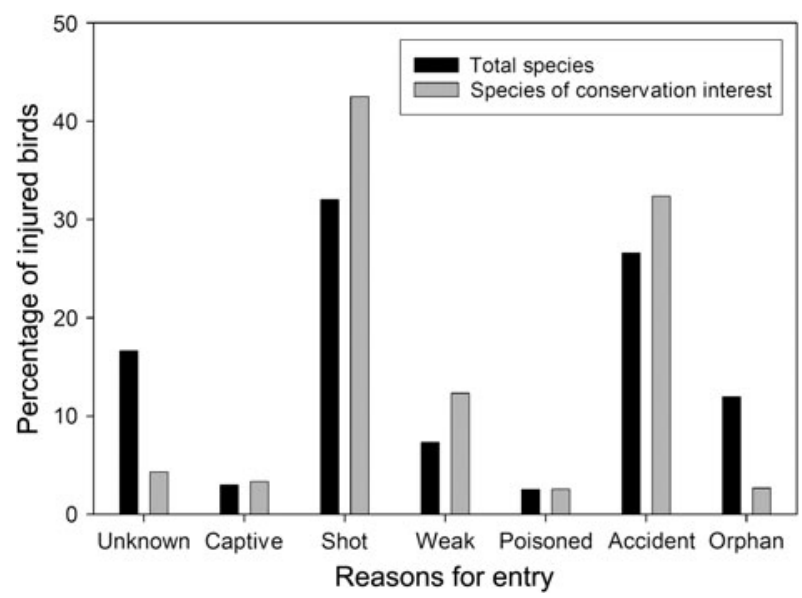

FIG. 3 Percentage of all birds and of birds of conservation interest in Europe received by the Hellenic Wildlife Hospital over 1996-2005, categorized according to the reason for entry.

\section{Results}

During the 10 years a total number of 21,190 individuals belonging to 469 bird species were admitted to the Hellenic Wildlife Hospital, with a marked increase from 1998 to 2000, and a general reduction thereafter (Fig. 2). Almost $15 \%$ ( 73 out of 469 ) of the species admitted to the Hospital are listed in the Birds Directive of the EU.

It was not possible to determine clearly the reasons for entry of $16 \%$ of the individuals received (Fig. 3). The greatest number of admissions (32\%) were due to shooting, with accidents and orphans/newborns the second and third most common reasons for admission (26 and $12 \%$ respectively; Fig. 3). The reasons for admission differed between raptors, migratory and aquatic species (Fig. 4). Gunshot wounds were the most frequent cause of injury for raptors

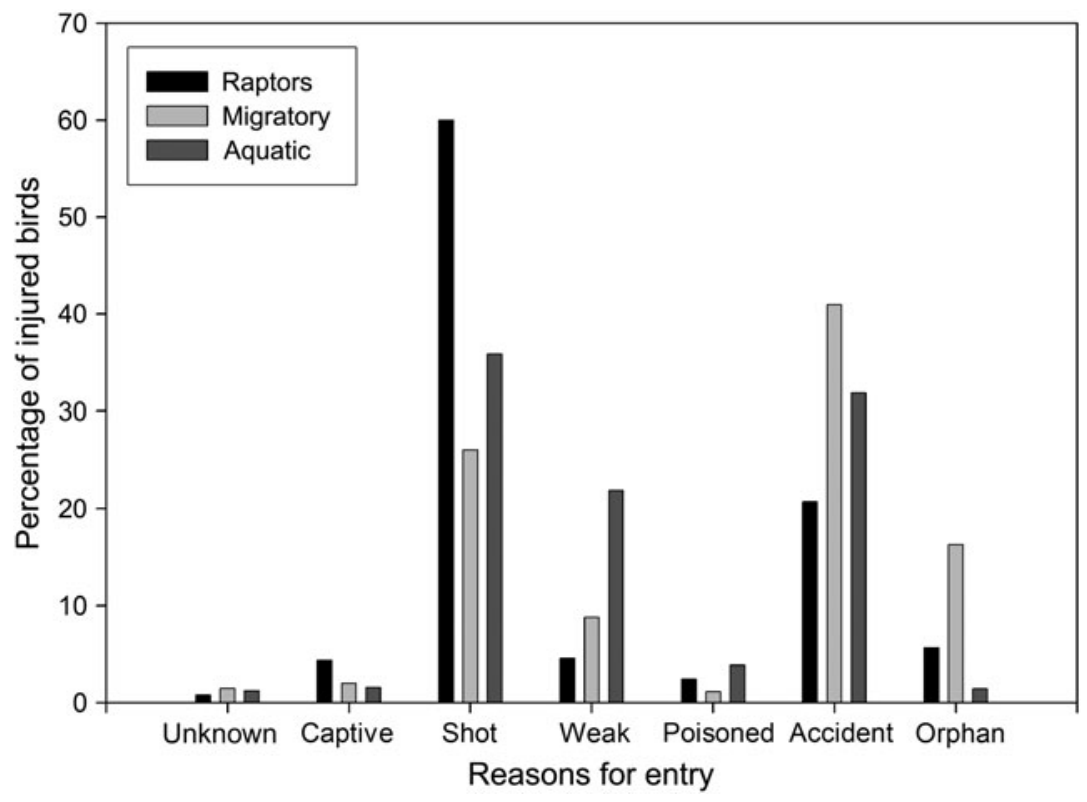

FIG. 4 Percentage of raptors and migratory and aquatic birds received by the Hellenic Wildlife Hospital over 1996-2005, categorized according to the reason for entry. 


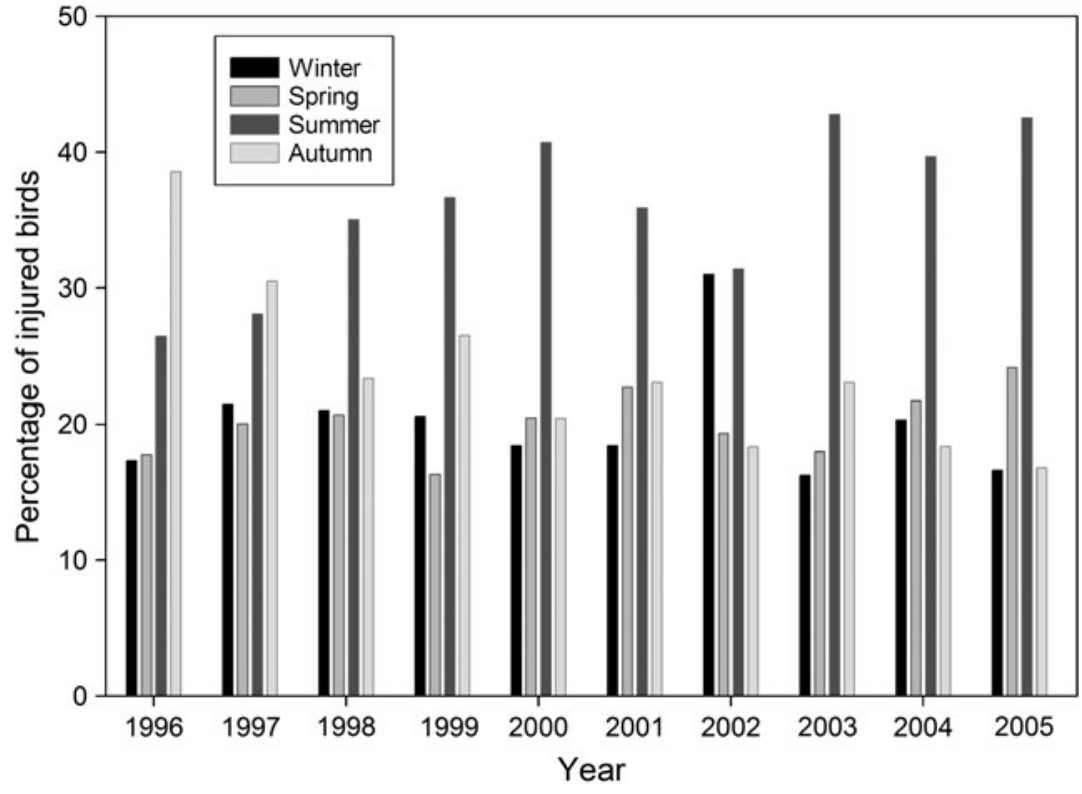

Fig. 5 Percentage of birds received, categorized by season, by the Hellenic Wildlife Hospital over 1996-2005.
$(60 \%)$ and aquatic (36\%) species, followed by accidents (raptors, 20\%; aquatic, 32\%). Accidents were the most frequent reason for injury of migratory (41\%) species followed by gunshots (26\%). Weak individuals and orphans were more frequently recorded among migratory and aquatic species, respectively, and less frequently for raptors. Shooting was the main cause of morbidity or mortality for species of high conservation importance, followed by accidents.

There were significant differences in the mean number of birds received per season (Kruskal-Wallis $H=8496.437$, $\mathrm{P}<0.001$ ), and the number of birds per season differed between years $\left(\chi^{2}=63.570, \mathrm{P}<0.001\right)$. The greatest number of injured birds were received during the summer
(Fig. 5). Except for the first 2 years, when autumn was the season with the highest contribution to the annual number of birds received, no clear seasonal pattern was apparent.

Multiple comparisons indicated significant differences in the number of shot birds received during winter and spring $\left(q=4.085, q_{\text {crit,o.05 }}=3.858\right)$, winter and summer $(q=5.653$, $\left.q_{\text {crit,o.05 }}=3.858\right)$ and autumn and summer $(q=3.922$, $\left.q_{\text {crit,o.05 }}=3.858\right)$. There were also significant differences in the number of cases recorded as accidents and in injured birds with undetermined cause of injury during summer and winter (accidents: $q=4.761, q_{\text {crit,o.05 }}=3.858$; unknown: $\left.q=6.438, q_{\text {crit,o.05 }}=3.858\right)$.

Ordination analysis demonstrated a clear association among zoogeographical regions regarding the taxonomic

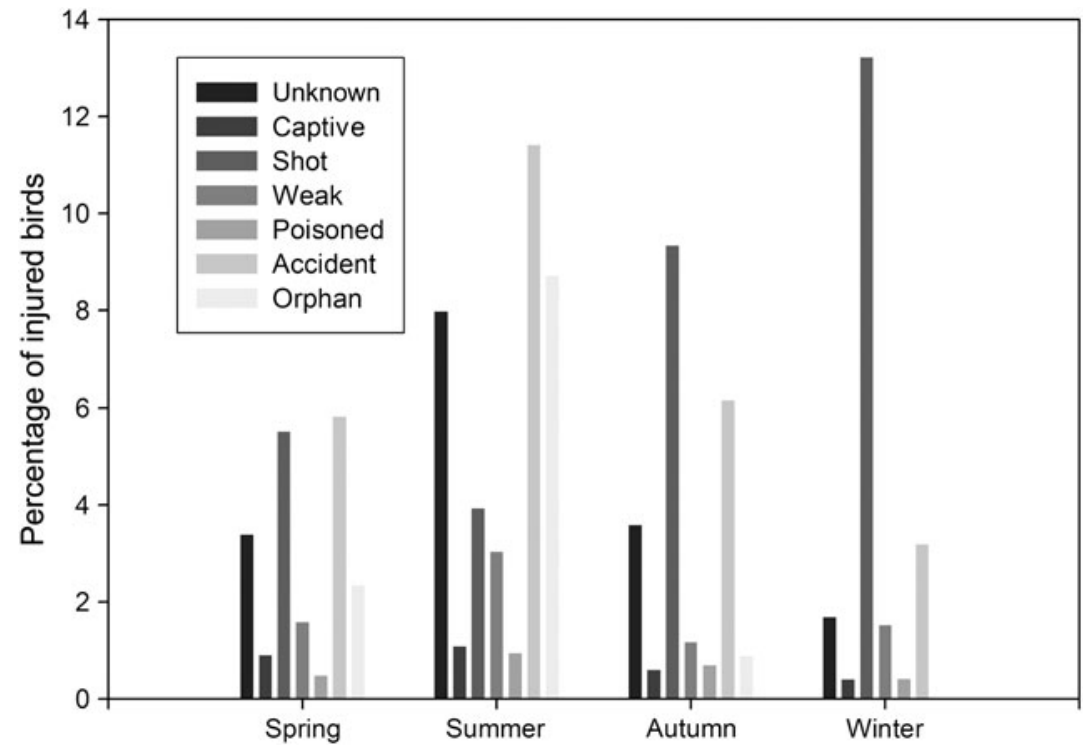

FIG. 6 Percentage of birds received by the Hellenic Wildlife Hospital per season (see text for details) over 1996-2005, categorized by reason for entry. 

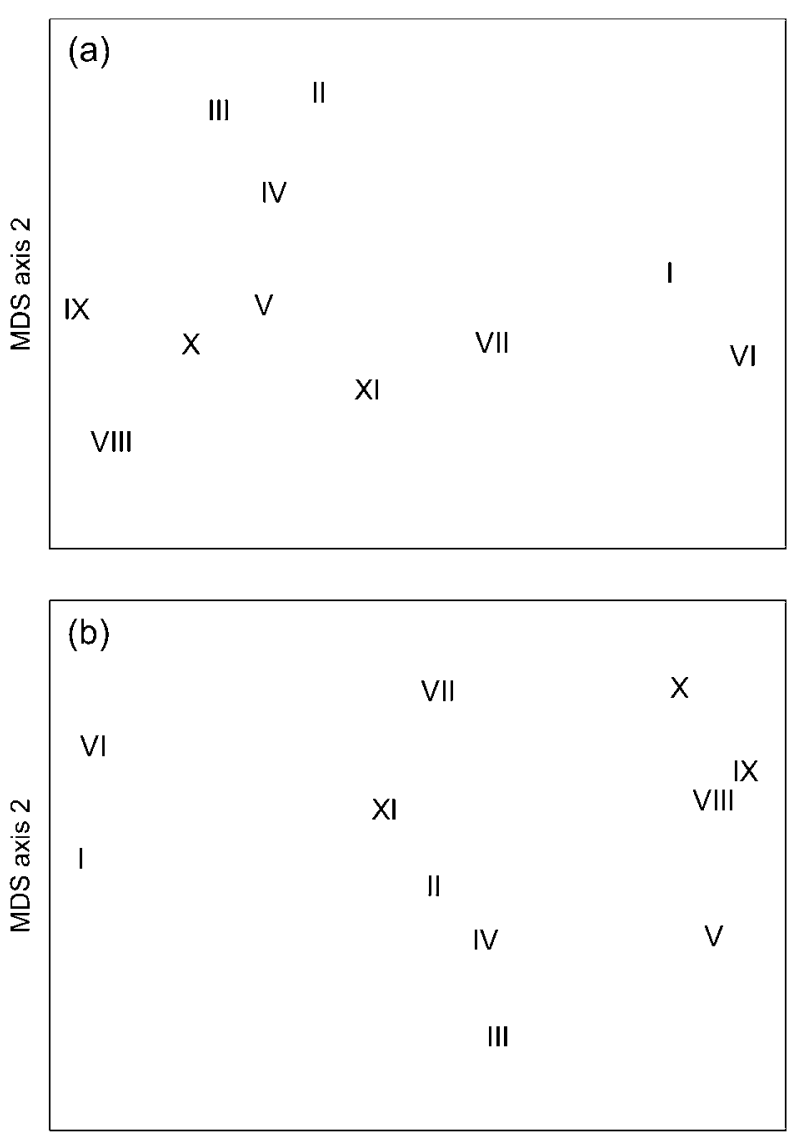

MDS axis 1

FIG. 7 Non-metric multidimensional scaling ordination plot (see text for further details) of zoogeographical regions (Fig. 1) based on (a) species composition of admissions, and (b) causes of mortality and morbidity of admissions. Bray-Curtis similarity was used for both ordinations. I, North-eastern Greece; II, North-western Greece; III, Heperus; IV, Thessaly; V, Ionian Islands; VI, Central Greece; VII, Peloponnesus; VIII, Cyclades; IX, Northern Aegean; X, Dodecanese; XI, Crete.

composition of species received at the centre (NMS stress o.01). A strong grouping occurs between North-eastern and Central Greece, which are clearly separated from other regions of the country (Fig. 7a).

The number of birds received differed significantly among the 11 zoogeographical regions (Kruskal-Wallis test, $H=73.288, \mathrm{P}<0.001$ ), with $>60 \%$ received from Northeastern and Central Greece. Ordination based on the causes of mortality or morbidity revealed a clear grouping among regions (NMS stress 0.04). There were three clusters (Fig. 7b). North-eastern and Central Greece were grouped together and clearly separated from the Ionian Islands, Cyclades, Dodecanese and Crete, which comprise marine areas of the country (Fig. 1). There was greater variation among the remaining regions, although they were grouped together. With the exception of North-eastern and Central Greece, in which most of the cases were due to accidents ( 35 and $39 \%$, respectively), shooting was the dominant reason for injury, contributing to $c .50 \%$ of the injuries in other regions $(41-72 \%)$.

\section{Discussion}

Data collected at rehabilitation centres could provide an additional tool to recognize wildlife threats because it allows the evaluation of the impact of several factors that are difficult to study directly. Our analysis has demonstrated that shooting was the main cause of injury to birds admitted to the Hellenic Wildlife Hospital during 19962005. Most shooting incidents occur during autumn and winter, which coincides with the hunting period in Greece (end of August to end of February), although shot birds were admitted throughout the year. However, the lack of significant differences in the number of shot birds between autumn and spring and spring and summer indicates that even though shooting is limited during the non-hunting period it is nevertheless an ongoing activity. This finding highlights the inefficiency of law enforcement against illegal shooting. In addition, the high number of shot birds admitted to the Hospital from throughout Greece during the non-hunting periods demonstrates that these breaches of the law are a national, rather than regional, phenomenon. The fact that several of the shot species are large bodied and have a characteristic flight (e.g. Ciconia nigra, Pelecanus onocrotalus), and are therefore easily identifiable, demonstrates the severity of illegal hunting.

Hunting regulations in Greece comply with European Union directives, international conventions and treaties for the protection of wildlife. These include the Bern Convention on Nature Protection, the Bonn Convention on the Conservation of Migratory Species of Wild Animals, the RAMSAR International Convention, the Habitat Directive of the European Council 92/43/EEC, the Birds Directive of the European Council 79/409/EEC, and CITES. Hunting is prohibited for all species included in the IUCN Red List (IUCN, 2007) and in the Birds Directive of the European Council. However, the inefficiency of law enforcement and the lack of compliance of hunting regulations with international conventions have been criticized (Hellenic Ornithological Society, 2006). The results of this study support such criticisms. In contrast to most European countries, where the location and spatial extent of hunting sites is explicitly determined, no private areas for hunting exist in Greece. Hunting is allowed at all public sites except for areas of particular interest (defined by the law on Measures for the Management of Wild Avifauna ref. no. 414985/29-11-85-O.G.R. 757/B'/18-12-85).

Fewer birds were admitted because of poisoning than for any other mortality factor. It is probable that poisoned birds die immediately and therefore only a limited number of poison victims are admitted to wildlife centres, leading to an underestimation of the significance of poisoning as 
a mortality factor (see also Mineau et al., 1999). Similar studies have also highlighted the relatively high contribution of orphaned young to the number of animals admitted to rehabilitation centres (Fix \& Barrows, 1990; Wendell et al., 2002).

Most of the birds admitted to the Hospital originated from North-eastern and Central Greece. This could be because of the higher density of people in this urbanized region, a higher degree of ecological awareness, or better facilities for shipping birds to the Hospital. The reduced admissions from other regions is most likely because of limited transportation options in rural areas and the expenses of shipping. Nevertheless, causes of morbidity and mortality varied between regions. Accidents were the most frequent cause of admission for animals received from North-eastern and Central Greece probably reflecting the increased human pressure in this area, which has a dense human population and busy transportation network.

The high number of shot raptors admitted during nonhunting periods provides clear evidence for the persecution of this group of species. Both gunshots and accidents were the main causes of injury to migratory and aquatic species, further demonstrating the significance of shooting as a major threat. The relatively high number of migratory and aquatic species admitted due to accidents may reflect increased public concern regarding specific species that could also be used as flagship species (sensu Bowen-Jones \& Entwistle, 2002). Public awareness programmes and research projects in Greece often focus on such species.

The types of injuries of animals submitted to rehabilitation centres are likely to be associated with human activities (Spalding \& Forrester, 1993) reflecting local conditions or threats (Casey \& Casey, 2000). This could produce biased evaluations of the causes of mortality or morbidity. We attempted to reduce this sampling bias by analysing data from all regions of the country (Brown \& Sleeman, 2002) but we acknowledge this limitation and suggest that complementary data from field surveys are also required. We consider the information collected at the Hellenic Wildlife Hospital to be a comprehensive data set for analysing threats for bird species in Greece. However, we caution that fluctuations in the number of individuals could reflect public awareness and concern (Brown \& Sleeman, 2002).

Habitat loss, fragmentation and degradation are critical factors affecting population viability but hunting has also led to extinction of several species in the past and is still one of the main threats to wildlife (BirdLife International, 2004). In addition to being a major source of mortality, hunting is also an important source of disturbance, affecting migratory behaviour and distribution of species (Fox \& Madsen, 1997; Béchet et al., 2003). However, large scale assessments of its impact on population sizes and composition are difficult to conduct because of practical difficulties. To estimate hunting pressure, data are collected by combining environmental and social surveys (Kaul et al., 2004; Johnson et al., 2005; Lehman et al., 2006). The collected information is often analysed with the use of a geographical information system and time series of satellite or aerial images (Ostwald, 2002; Campbell, 2005), although the applicability of such combined methods is limited to local studies.

There is an urgent need for conservation actions to conserve the bird populations of Greece. Actions need to focus on strengthening the enforcement of existing regulations. We are preparing a detailed report for submission to the Greek governmental agencies responsible for hunting regulations. The results presented will provide evidence highlighting the need for strict enforcement of the existing legislation for protecting birds and closer compliance with EU regulations.

\section{Acknowledgements}

We thank Maria Zomeni and Joseph Tzanopoulos for constructive comments on the manuscript. We wish to thank all volunteers who participated in the 1996-2005 projects of the Hellenic Wildlife Hospital. We thank Panoriou Euegenia and Galinos Spyros for their help with data processing.

\section{References}

BARTEN, S.L. (1986) Common problems among reptiles presented to rehabilitation centres. Wildlife Rehabilitation, 5, 80-85.

Béchet, A., Giroux, J., Gauthier, G., Nichols, D.J. \& Hines, E.J. (2003) Spring hunting changes the regional movements of migrating greater snow geese. Journal of Applied Ecology, 40, $553-564$.

BirdLife International (2004) Threatened Birds of the World, CD-ROM. BirdLife International, Cambridge, UK.

Bowen-Jones, E. \& Entwistle, A. (2002) Identifying appropriate flagship species: the importance of culture and local contexts. Oryx, 36, 189-195.

Brown, D.J. \& Sleeman, M.J. (2002) Morbidity and mortality of reptiles admitted to the wildlife center of Virginia, 1991 to 2000. Journal of Wildlife Diseases, 38, 699-705.

Burton, D.L. \& Dufour, K.A. (2000) An overview of urban wildlife morbidity and mortality in the Midwest. Wildlife Rehabilitation, 18, 77-108.

Campbell, O.M. (2005) The ecological and social context of mammal hunting in the coastal savanna of Ghana. Geoforum, 36, 667-680.

Casey, A.M. III \& CASey, S.J. (2000) A survey of conditions seen in wildlife admitted for wildlife rehabilitation. Wildlife Rehabilitation, 18, 143-160.

Clarke, K.R. \& Gorley, R.N. (2001) PRIMER v5: User Manual/ Tutorial. Plymouth Marine Laboratory, PRIMER-E Ltd, Plymouth, UK.

Clumpner, J.C., Hansen, J.M. \& Borgia, A.L. (2000) Annotated Bibliography of Wildlife Rehabilitation. Second edition, 19822000. National Wildlife Rehabilitators Association, St. Cloud, USA. 
Dafis, S., Papstergiadou, E., Georgiou, K., Mpampalonas, D., Georgiadis, T., Papageorgiou, M. et al. (1997) Directive 92/43/EEC. The Greek “Habitat” Project Natura 2000: An Overview. Commission of the European Community DG XCI, The Goulandris Natural History Museum-Hellenic Biotope/Wetland Centre, Thessaloniki, Greece.

Deem, S.L., Terrell, S.P. \& Forrester, D.J. (1998) A retrospective study of morbidity and mortality of raptors in Florida: 1988-1994. Journal of Zoo and Wildlife Medicine, 29, 160-164.

FIX, A.S. \& B ARRows, S.Z. (1990) Raptors rehabilitated in Iowa during 1986 and 1987: a retrospective study. Journal of Wildlife Diseases, 26, 18-21.

Fox, A.D. \& Madsen, J. (1997) Behavioural and distributional effects of hunting disturbance on waterbirds in Europe: implications for refuge design. Journal of Applied Ecology, 34, 1-13.

Hellenic Ornithological Society (2006) Announcement of Hunting Regulation for 2006-2007. Http://www.ornithologiki.gr/gr/hos/dt/ dt_20060804.htm [accessed 12 March 2007].

International Wildlife Rehabilitation Council (2007) Conserving and Protecting Wildlife and Habitat through Wildlife Rehabilitation. Http://www.iwrc-online.org [accessed 10 March 2007].

IUCN (2007) 2007 IUCN Red List of Threatened Species. IUCN, Gland, Switzerland. Http://www.iucnredlist.org [accessed 28 September 2007].

Johnson, A., Singh, S., Duangdala, M. \& Hedemark, M. (2005) The western black crested gibbon Nomascus concolor in Laos: new records and conservation status. Oryx, 39, 311-317.

Jones, M.P. (1999) Selected infectious diseases of birds of prey. Wildlife Rehabilitation, 17, 27-36.

Kaul, R., Hilaluddin, Jandrotia, J.S. \& McGowan, P.J.K. (2004) Hunting of large mammals and pheasants in the Indian western Himalayas. Oryx, 38, 426-431.

Kuehnelt, W. (1965) Tenebrionidae. In Catalogous Faune Graeciae. Pars 1. (ed. A. Kanellis), pp. 1-6o. To Wuno, Thessaloniki, Greece.

Lehman, S.M., Ratsimbazafy, J., Rajaonson, A. \& Day, S. (2006) Decline of Propithecus diadema edwardsi and Varecia variegata variegata (Primates: Lemuridae) in south-east Madagascar. Oryx, 40, 108-111.

Leston, F.V.L., Amanda, D. \& Rodewald, D.A. (2006) Are urban forests ecological traps for understorey birds? An examination using Northern cardinals. Biological Conservation, 131, 566-574.

Letcher, H.B., Priddy, A.J., Walters, R.J. \& Crowder, B.L. (1998) An individual-based, spatially-explicit simulation model of the population dynamics of the endangered red-cockaded woodpecker, Picoides borealis. Biological Conservation, 86, 1-14.

Lunney, D., Gresser, M.S., Mahon, P.S. \& Matthews, A. (2004) Post-fire survival and reproduction of rehabilitated and unburnt koalas. Biological Conservation, 120, 567-575.

MignuCCi-Giannoni, A.A. (1999) Assessment and rehabilitation of wildlife affected by an oil spill in Puerto Rico. Environmental Pollution, 104, 323-333.

Miller, E.A. (1997). Common diseases and injuries of wild urban songbirds. Wildlife Rehabilitation, 15, 109-117.

Mineau, P., Fletcher, M.R., Glaser, L.C., Thomas, N.J., Brassard, C., Wilson, L.K. et al. (1999) Poisoning of raptors with organophoshorus and carbamate pesticides with emphasis on Canada, US and UK. Journal of Raptor Research, 33, 1-37.

Mishra, C., Madhusudan, M.D. \& Datta, A. (2006) Mammals of the high altitudes of western Arunachal Pradesh, eastern Himalaya: an assessment of threats and conservation needs. Oryx, 40, 29-35.

Morishita, T.Y., Fullerton, A.T., Lowenstine, L.J., Gardner, I.A. \& BRooks, D.L. (1998) Morbidity and mortality in free-living raptorial birds of northern California: a retrospective study. Journal of Avian Medicine and Surgery, 12, 78-81.
Ostwald, M. (2002) GIS-based support tool system for decisionmaking regarding local forest protection: illustrations from Orissa, India. Environmental Management, 30, 35-45.

Parsons, J.N., Calf, M.K., Underhill, G.L. \& Strauss, V. (2006) The rehabilitation of swift terns Sterna bergii incapacitated by marine foam on Robben Island, South Africa. Ostrich, 77, 95-98.

Parsons, J.N. \& Underhill, G.L. (2005) Oiled and injured African penguins Spheniscus demersus and other seabirds admitted for rehabilitation in the Western Cape, South Africa, 2001 and 2002. African Journal of Marine Science, 27, 289-296.

Porter, J.H. \& McEntyre, A. (1988) Deformities in San Juan Islands' Columbia black tailed deer (Odocoileus hemionus columbianus). Wildlife Rehabilitation, 7, 47-56.

Pullin, A.S. (2002) Conservation Biology. Cambridge University Press, Cambridge, UK.

RijKsen, D.H. (1974) Orang-utan conservation and rehabilitation in Sumatra. Biological Conservation, 6, 20-25.

SCHulz, T.A. (1986) The conservation and rehabilitation of the common barn owl. Wildlife Rehabilitation, 5, 146-168.

Soulé, M. \& Orians, G.H. (2001) Conservation Biology: Research Priorities for the Next Decade, 2nd edition. Island Press, Washington, DC, USA.

Spalding, M.G. \& Forrester, D.J. (1993) Disease monitoring of free-ranging and released wildlife. Journal of Zoo and Wildlife Medicine, 24, 271-280.

Sullivan, J.B., Reid, A.T. \& Bugoni, L. (2006) Seabird mortality on factory trawlers in the Falkland Islands and beyond. Biological Conservation, 131, 495-504.

TsenG, S.F. (1999) Considerations in care for birds affected by oil spills. Seminars in Avian and Exotic Pet Medicine, 8, 21-31.

Underhill, L.G., Bartlett, P.A., Baumann, L., Crawford, R.J.M., Dyer, B.M., Gildenhuys, A. et al. (1999) Mortality and survival of African penguins Spheniscus demersus involved in the Apollo Sea oil spill: an evaluation of rehabilitation efforts. Ibis, 141, 29-37.

Wemmer, C.L., Özesmi, U. \& Cuthiert, J.F. (2001) A habitatbased population model for the Great Lakes population of the piping plover (Charadrius melodus). Biological Conservation, 99, $169-181$.

Wendell, D.M., Sleeman, M.J. \& Kratz, G. (2002) Retrospective study of morbidity and mortality of raptors admitted to Colorado state university veterinary teaching hospital during 1995 to 1998. Journal of Wildlife Diseases, 38, 101-106.

Willemse, F. (1984) Catalogue of the Orthoptera of Greece. Fauna Graeciae I. Hellenic Zoological Society, Athens, Greece.

Zar, J.H. (1999) Biostatistical Analysis, 4th edition. Prentice Hall, New Jersey, USA.

\section{Biographical sketches}

Antonios Mazaris is carrying out research on conservation biology, reserve selection, population dynamics and ecological risk assessment. YIANNIS MAMAKIS works with the management of the Hellenic Wildlife Hospital and conducts research on the ecology, behaviour and biology of various bird species. STAUROS KALPAKIS has worked on various conservation issues in Greece since the 1980s, with a particular focus on the ecology and behaviour of bird species, and is currently working on the establishment of protected areas for birds. Yiannis Poulopoulos established the Hellenic Wildlife Hospital in 1984. He participates in conservation and monitoring programmes for bird species throughout Greece. YianNis MATSINos has a particular interest in the ecology and population dynamics of birds. 